\title{
DIGITALCOMMONS
}

$@$ WAYNESTATE-

Wayne State University

$10-1-2003$

\section{Dirichlet Boundary Control of Hyperbolic Equations in the Presence of State Constraints}

Boris S. Mordukhovich

Wayne State University, boris@math.wayne.edu

Jean-Pierre Raymond

Wayne State University

\section{Recommended Citation}

Mordukhovich, Boris S. and Raymond, Jean-Pierre, "Dirichlet Boundary Control of Hyperbolic Equations in the Presence of State Constraints" (2003). Mathematics Research Reports. Paper 15.

http://digitalcommons.wayne.edu/math_reports/15

This Technical Report is brought to you for free and open access by the Mathematics at DigitalCommons@WayneState. It has been accepted for inclusion in Mathematics Research Reports by an authorized administrator of DigitalCommons@WayneState. 


\section{DIRICHLET BOUNDARY CONTROL OF HYPERBOLIC EQUATIONS IN THE PRESENCE OF STATE CONSTRAINTS}

RORIS S MORDUKHOVICH and JEAN-PIERRE RAYMOND

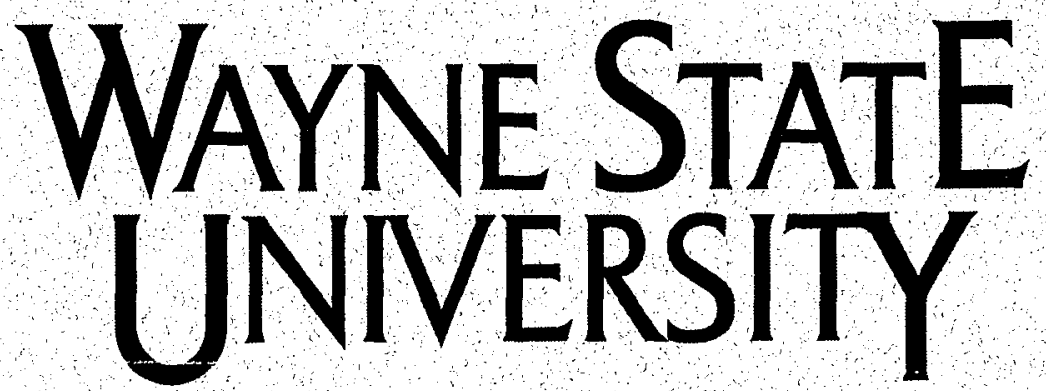

Detroit, MI 48202

Department of Mathematics

Research Report

2003 Series

\#10

This research was partly supported by the National Science Foundation. 


\title{
DIRICHLET BOUNDARY CONTROL OF HYPERBOLIC EQUATIONS IN THE PRESENCE OF STATE CONSTRAINTS
}

\author{
BORIS S. MORDUKHOVICH ${ }^{1}$ and JEAN-PIERRE RAYMOND ${ }^{2}$
}

\begin{abstract}
We study optimal control problems for hyperbolic equations (focusing on the multidimensional wave equation) with control functions in the Dirichlet boundary conditions under hard/pointwise control and state constraints. Imposing appropriate convexity assumptions on the cost integral functional, we establish the existence of optimal control and derive new necessary optimality conditions in the integral form of the Pontryagin Maximum Principle for hyperbolic state-constrained systems.
\end{abstract}

Key Words. Optimal control, Hyperbolic equations, Dirichlet boundary controls, State constraints, Integral maximum principle.

AMS Classification. Primary 49K20, 49J20, Secondary 93C20, 35L20.

\section{Introduction}

This paper is devoted to the study of optimal control problems for state-constrained hyperbolic equations with controls in Dirichlet boundary conditions. We pay the main attention to the following problem governed by multidimensional wave equation. Given an open bounded domain $\Omega \subset \mathbb{R}^{N}$ with the boundary $\Gamma$ of class $C^{2}$, we consider the problem of minimizing the integral functional

$$
J(y, u)=\int_{\Omega} \phi(x, y(T)) d x+\int_{Q} g(x, t, y) d x d t+\int_{\Sigma} h(s, t, u) d s d t
$$

\footnotetext{
${ }^{1}$ Department of Mathematics, Wayne State University, Detroit, MI 48202, USA, boris@math.wayne.edu. Research of the first author was partly supported by the National Science Foundation under grants DMS-0072179 and DMS-0304989.

${ }^{2}$ Laboratoire MIP, Université Paul Sabatier, 31062 Toulouse Cedex 4, France, raymond@mip.upstlse.fr
} 
for a fixed time $T>0$ over admissible pairs $(y, u)$ satisfying the wave equation with control in the Dirichlet boundary conditions

$$
\begin{cases}y_{t t}-\Delta y=f & \text { in } Q:=\Omega \times] 0, T[ \\ y=u & \text { on } \Sigma:=\Gamma \times] 0, T[ \\ y(0)=y_{0}, \quad y_{t}(0)=y_{1} & \text { in } \Omega\end{cases}
$$

subject to the pointwise control and state constraints

$$
u \in U_{a d} \subset L^{2}(\Sigma), \quad y \in \mathcal{C} \subset C\left([0, T] ; L^{2}(\Omega)\right)
$$

where $f \in L^{1}\left(0, T ; H^{-1}(\Omega)\right), y_{0} \in L^{2}(\Omega)$, and $y_{1} \in H^{-1}(\Omega)$ are given functions. This optimal control problem is shortly described by

$$
\inf \left\{J(y, u) \mid(y, u) \text { satisfies (1.1), } u \in U_{a d}, \quad y \in \mathcal{C}\right\}
$$

The assumptions on the initial data of $(P)$ will be listed and discussed in Section 2.

It is well known that optimal control problems with pointwise state constraints belong to one of the most challenging and difficult classes in control theory. Quite recently, growing interest to such problems for parabolic equations has been taken in $[2,3,4,6,11,12,13]$; see also the references therein. Much less has been done for hyperbolic systems. Some control problems for the wave equation in the presence of state constraints are considered in $[5,15,14]$ for distributed controls. We are not familiar with any results on boundary control problems for the wave equation and/or for other partial differential equations of the hyperbolic type.

Note that there are essential differences between parabolic and hyperbolic systems. One of the most principal one is that hyperbolic equations generally exhibit less regularity. It is well known, in particular, that solutions $y$ to the state equation (1.1) only belong to the space $C\left([0, T] ; L^{2}(\Omega)\right.$ ) (which makes the state constraints $y \in \mathcal{C} \subset C\left([0, T] ; L^{2}(\Omega)\right)$ to be meaningful) in compare with much higher regularity in the case of parabolic equations; see, e.g., $[9,11]$. We refer the reader to $[5,7,8,10]$ and their bibliographies for more discussions on other important differences between parabolic and hyperbolic systems. The lack of regularity does not allow one to apply to 
hyperbolic boundary control problems the methods developed in the mentioned papers for the case of parabolic equations.

In this paper we use a different strategy to derive necessary optimality conditions for the hyperbolic state-constrained problem $(P)$. Our approach is based on a reduction of the original control problem $(P)$ to an abstract optimization problem in Banach spaces with operator and geometric constraints of a special type corresponding to the structure of the given control problem. Then we apply to the abstract optimization/abtract control problem a version of the Lagrange multiplier rule established in [1]. The main task is to expressed the requirements of the abstract multiplier rule in terms of the initial data of the hyperbolic Dirichlet boundary control problem $(P)$. To furnish this, we employ delicate regularity results for hyperbolic systems obtained in [7]. The lack of regularity, in comparison with parabolic systems, is compensated by extra convexity assumptions. Indeed, we impose convexity of the functions in the integral cost functional with respect to both control and state variables, which is not requires in the parabolic case; cf. [11, 12]. The assumptions made and the available regularity allow us to establish also the existence theorem for optimal controls in the original problem. Note that, although we present the main results only in the case multidimensional wave equation for the hyperbolic dynamics, the results obtained can be extended to more general hyperbolic equations with a strongly elliptic operator instead of the Laplacian. This can be done in a similar way based on the comprehensive treatment of regularity issues for nonhomogeneous Dirichlet boundary-value problems conducted in the seminal paper by Lasiecka, Lions, and Triggiani [7] for second-order hyperbolic equations.

The rest of the paper is organized as follows. Section 2 contains the basis assumptions imposed on the initial data and the formulation of the main results of the paper, which establish the existence of optimal controls and necessary optimality conditions obtained in the integral form of the Pontryagin Maximum Principle for the state-constrained hyperbolic system. Section 3 is devoted to the appropriate notion of weak solutions to the hyperbolic state equation with Dirichlet boundary conditions, the existence and uniqueness of which is guaranteed by the regularity results of [7]. In this section we also present the proof of the existence theorem for optimal controls in the optimization problem under consideration. 
The remaining two sections concern the proof of necessary optimality conditions. A crucial part of this proof is the variational analysis of the adjoint system, the solution of which is understood in the appropriate weak sense. This is conducted in Section 4. The concluding Section 5 is based on this analysis and the hyperbolic regularity properties, which allow us to deduce the Pontryagin-type necessary optimality conditions for the original problem from a version of the Lagrange Multiplier Rule in an auxiliary abstract optimization/control problem in suitable Banach spaces.

\section{Basic Assumptions and Main Results}

Let us first recall some Notation that is mostly standard in this area. Denote by $\mathcal{M}\left([0, T] ; L^{2}(\Omega)\right)$ the space of measures on $[0, T]$ with values in $L^{2}(\Omega)$, which is the topological dual of $C\left([0, T] ; L^{2}(\Omega)\right)$. The topological dual of

$$
\left.\left.C_{0}(] 0, T\right] ; L^{2}(\Omega)\right):=\left\{y \in C\left([0, T] ; L^{2}(\Omega)\right) \mid y(0)=0\right\}
$$

is denoted by $\left.\left.\mathcal{M}_{b}(] 0, T\right] ; L^{2}(\Omega)\right)$. It is well known that $\left.\left.\mathcal{M}_{b}(] 0, T\right] ; L^{2}(\Omega)\right)$ can be identified with the subspace of $\mathcal{M}\left([0, T] ; L^{2}(\Omega)\right)$ of measures $\mu \in \mathcal{M}\left([0, T] ; L^{2}(\Omega)\right)$ such that $\left.\mu\right|_{\Omega \times\{0\}}=0$, where $\left.\mu\right|_{\Omega \times\{0\}}$ denotes the restriction of $\mu$ to $\Omega \times\{0\}$. The same kinds of notation are used throughout the paper for other similar spaces. For $z \in L^{2}(Q)$ we denote by $z_{t}$ (respectively by $z_{t t}$ ) the derivative (respectively the second derivative) of $z$ in the $t$-variable, in the sense of distributions on $Q$.

Given a Banach space $Z$, the canonical duality pairing between $Z$ and $Z^{*}$ is denoted by $\langle\cdot,\rangle_{Z \times Z^{*}}$. For example, the duality pairing between $\left.C_{0}(10, T] ; L^{2}(\Omega)\right)$ and $\left.\left.\mathcal{M}_{b}(] 0, T\right] ; L^{2}(\Omega)\right)$ is denoted by $\langle\cdot, \cdot\rangle_{\left.\left.\left.C_{0}(j 0, T] ; L^{2}(\Omega)\right) \times \mathcal{M}_{b}(] 0, T\right] ; L^{2}(\Omega)\right)}$. If $y$ belongs to $C\left([0, T] ; L^{2}(\Omega)\right)$ and $\mu$ belongs to $\left.\left.\mathcal{M}_{b}(] 0, T\right] ; L^{2}(\Omega)\right)$, we still use the notation

$$
\langle y, \mu\rangle_{\left.C\left([0, T] ; L^{2}(\Omega)\right) \times \mathcal{M}_{b}(j 0, T] ; L^{2}(\Omega)\right)} \quad \text { for } \quad\langle y, \tilde{\mu}\rangle_{C\left([0, T] ; L^{2}(\Omega)\right) \times \mathcal{M}\left([0, T] ; L^{2}(\Omega)\right)}
$$

where $\tilde{\mu}$ is the extension of $\mu$ by zero to $\Omega \times\{0\}$. The same abuse of notation is used for a measure $\mu$ belonging to $\mathcal{M}_{b}(] 0, T\left[; L^{2}(\Omega)\right)$. When there is no ambiguity, we sometimes write $\langle\cdot, \cdot\rangle$ in place of $\langle\cdot, \cdot\rangle_{Z \times Z^{*}}$.

Since it is important to specify an appropriate regularity of solutions to boundaryvalue problems for the equations considered, we often use expressions of the type $\left(y, y_{t}\right) \in C\left([0, T] ; L^{2}(\Omega)\right) \times C\left([0, T] ; H^{-1}(\Omega)\right)$ is a solution to $(1.1)$, in place of 
$y$ is the solution to (1.1).

Next let us formulate the Basic Assumptions imposed on the initial data of the optimal control problem $(P)$.

(A1) For every $y \in \mathbb{R}, \phi(\cdot, y)$ is measurable in $\Omega$ and $\phi(\cdot, 0)$ belongs to $L^{1}(\Omega)$. For almost every (a.e.) $x \in \Omega, \phi(x, \cdot)$ is a continuous, nonnegative, and convex function on $\mathbb{R}$.

(A2) For every $y \in \mathbb{R}, g(\cdot, \cdot, y)$ is measurable in $Q$ and $g(\cdot, \cdot, 0)$ belongs to $L^{1}(Q)$. For a.e. $(x, t) \in Q, g(x, t, \cdot)$ is a continuous, nonnegative, and convex function on $\mathbb{R}$.

(A3) For every $u \in \mathbb{R}, h(\cdot, u)$ is measurable on $\Sigma$ and $h(\cdot, 0)$ belongs to $L^{1}(\Sigma)$. For almost every $(s, t) \in \Sigma, h(s, t, \cdot)$ is a continuous and convex function on $\mathbb{R}$. Moreover $h$ satisfies the following growth condition

$$
|u|^{2} \leq h(s, t, u)
$$

(A4) The set $\mathcal{C}$ is a closed and convex subset of $C\left([0, T] ; L^{2}(\Omega)\right)$ with nonempty interior, and $U_{a d}$ is a closed and convex subset of $L^{2}(\Sigma)$. We also suppose that the function $(x, t) \mapsto y_{0}(x)$ belongs to the interior of $\mathcal{C}$ and that there is $u \in U_{a d}$ satisfying $y_{u} \in \mathcal{C}$ and $J\left(y_{u}, u\right)<\infty$ for the corresponding solution $y_{u}$ to the Dirichlet problem (1.1).

(A5) For a.e. $x \in \Omega, \phi(x, \cdot)$ is of class $C^{1}$ satisfying

$$
\left|\phi_{y}^{\prime}(x, y)\right| \leq C(1+|y|) \text { for some constant } \quad C>0
$$

(A6) For a.e. $(x, t) \in Q, g(x, t, \cdot)$ is of class $C^{1}$ satisfying

$$
\left|g_{y}^{\prime}(x, t, y)\right| \leq C(1+|y|) \text { for some constant } \quad C>0
$$

(A7) For a.e. $(s, t) \in \Sigma, h(s, t, \cdot)$ is of class $C^{1}$ satisfying

$$
\left|h_{u}^{\prime}(s, t, u)\right| \leq C(1+|u|) \quad \text { for some constant } \quad C>0
$$

The assumptions made above seem to be natural for the optimal control problem under consideration. Probably the most restrictive assumptions involve the convexity of the integrands in the cost functional with respect to both control and state variables. While the convexity with respect to control variables happens to be unavoidable from viewpoints of the general existence theory in optimal control and variational analysis 
involving weak convergences of control functions, the additional convexity with respect to state variables looks to be a specific feature of Dirichlet boundary control problems for hyperbolic equations to compensate the lack of regularity.

Now we are ready to formulate the main results of the paper: the existence theorem and necessary optimality conditions. Note that the notions of solutions to the state and adjoint equations needed for these results will be rigorously clarified in Sections 3 and 4 , respectively.

Theorem 2.1 (existence of optimal controls). Suppose that assumptions (A1)(A4) are satisfied. Then the optimal control problem $(P)$ admits an optimal solution.

The proof of Theorem 2.1 is given in Section 3.

Theorem 2.2 (necessary optimality conditions). Suppose that assumptions (A1)$(A 7)$ are satisfied. Then for every optimal solution $(\bar{y}, \bar{u})$ to problem $(P)$ the following conditions hold: there are $\lambda \in \mathbb{R}^{+}$and $\left.\left.\mu \in \mathcal{M}_{b}(] 0, T\right] ; L^{2}(\Omega)\right)$ such that

$$
\begin{gathered}
(\lambda, \mu) \neq 0, \quad\langle\mu, z-\vec{y}\rangle \leq 0 \quad \text { for all } z \in \mathcal{C} \\
\int_{\Sigma}\left(\frac{\partial p}{\partial \nu}+\lambda h_{u}^{\prime}(s, t, \vec{u})\right)(u-\bar{u}) d s d t \geq 0 \quad \text { for all } u \in U_{a d}
\end{gathered}
$$

where $p$ is the corresponding solution to the adjoint system

$$
\begin{aligned}
& \begin{cases}p_{t t}-\Delta p=\lambda g_{y}^{\prime}(x, t, \bar{y})+\left.\mu\right|_{Q} & \text { in } Q=\Omega \times] 0, T[, \\
p=0 & \text { on } \Sigma=\Gamma \times] 0, T[,\end{cases} \\
& p(T)=y_{0}, \quad p_{t}(T)=-\lambda \phi_{y}^{\prime}(x, \bar{y}(T))-\left.\mu\right|_{\Omega \times\{T\}} \quad \text { in } \Omega . \\
& \text { Moreover, if there exists }(y, u) \in Y \times\left(U_{a d}-\bar{u}\right) \text { satisfying } \\
& \left\{\begin{array}{l}
y_{t t}-\Delta y=0 \text { in } Q, \quad y=u \text { on } \Sigma, \\
y(0)=0, \quad y_{t}(0)=0 \text { in } \Omega,
\end{array} \quad \text { and } \bar{y}+y \in \operatorname{int} \mathcal{C},\right.
\end{aligned}
$$

then one can take $\lambda=1$ in (2.2)-(2.3).

Note that the integral condition (2.2) is formulated as a part of the minimum (not maximum) principle, which is more convenient in our framework. The proof of Theorem 2.2 is given in Section 5 with the preliminary analysis of the adjoint system conducted in Section 4. 


\section{Regularity of Weak Solutions and Existence of Optimal Controls}

Let us first recall the definition of solutions to the nonhomogeneous Dirichlet boundary problem (1.1) for the wave equation appropriate to the purposes of this paper. The following notion of weak solutions meets our requirements.

Definition 3.1 (weak solutions to the original system). A function $\left(y, y_{t}\right) \in$ $C\left([0, T] ; L^{2}(\Omega)\right) \times C\left([0, T] ; H^{-1}(\Omega)\right)$ is a WEAK SOLUTION to (1.1) if one has

$$
\begin{aligned}
& \int_{Q} f z d x d t=\int_{Q} y \varphi d x d t+\left\langle y_{t}(T), z^{0}\right\rangle_{H^{-1}(\Omega) \times H_{0}^{1}(\Omega)} \\
& -\left\langle y_{t}(0), z(0)\right\rangle_{H^{-1}(\Omega) \times H_{0}^{1}(\Omega)}-\int_{\Omega} y(T) z^{1} d x+\int_{\Omega} y(0) z_{t}(0) d x+\int_{\Sigma} \frac{\partial z}{\partial \nu_{u}} d s d t
\end{aligned}
$$

for all $\left(\varphi, z^{0}, z^{1}\right) \in L^{1}\left(0, T ; L^{2}(\Omega)\right) \times H_{0}^{1}(\Omega) \times L^{2}(\Omega)$, where $z$ solves the homogeneous Dirichlet problem

$$
\begin{cases}z_{t t}-\Delta z=\varphi & \text { in } Q, \\ z=0 & \text { on } \Sigma, \\ z(T)=z^{0}, \quad z_{t}(T)=z^{1} & \text { in } \Omega .\end{cases}
$$

The importance of the defined notion of weak solutions to the hyperbolic system (1.1) is due to the following fundamental regularity result established in [7, Theorem 2.3], which ensures the existence, uniqueness, and continuous dependence of weak solutions to (1.1) on the initial and boundary conditions in appropriate Banach spaces.

Theorem 3.2 (basic regularity). For every $\left(f, u, y_{0}, y_{1}\right) \in L^{1}\left(0, T ; H^{-1}(\Omega)\right) \times L^{2}(\Sigma) \times$ $L^{2}(\Omega) \times H^{-1}(\Omega)$ the Dirichlet problem (1.1) admits a unique weak solution $\left(y, y_{t}\right)$ in $C\left([0, T] ; L^{2}(\Omega)\right) \times C\left([0, T] ; H^{-1}(\Omega)\right)$. Moreover, the mapping $\left(f, u, y_{0}, y_{1}\right) \mapsto\left(y, y_{t}\right)$ is linear and continuous from $L^{1}\left(0, T ; H^{-1}(\Omega)\right) \times L^{2}(\Sigma) \times L^{2}(\Omega) \times H^{-1}(\Omega)$ into $C\left([0, T] ; L^{2}(\Omega)\right) \times C\left([0, T] ; H^{-1}(\Omega)\right)$.

Theorem 3.2 pays a crucial role in further considerations of the paper. This theorem suggests us to introduce the space of admissible state functions, that is the space of 
solutions to system $(1.1)$ when $\left(f, u, y_{0}, y_{1}\right) \in L^{1}\left(0, T ; H^{-1}(\Omega)\right) \times L^{2}(\Sigma) \times L^{2}(\Omega) \times$ $H^{-1}(\Omega)$, as follows

$$
\begin{aligned}
Y:=\{ & y \in C\left([0, T] ; L^{2}(\Omega)\right) \mid y_{t} \in C\left([0, T] ; H^{-1}(\Omega)\right) \\
& \left.y_{t t}-\Delta y \in L^{1}\left(0, T ; H^{-1}(\Omega)\right),\left.y\right|_{\Sigma} \in L^{2}(\Sigma)\right\} .
\end{aligned}
$$

Endowed with the norm

$$
y \mapsto\|y\|_{C\left([0, T) ; L^{2}(\Omega)\right)}+\left\|y_{t}\right\|_{C\left([0, T] ; H^{-1}(\Omega)\right)}+\|y t t-\Delta y\|_{L^{1}\left(0, T ; H^{-1}(\Omega)\right)}+\left\|\left.y\right|_{\Sigma}\right\|_{L^{2}(\Sigma)}
$$

$Y$ is a Banach space.

Now based on Theorem 3.2 and standard results on the lower semicontinuity of integral functionals in weak topologies under the assumptions made, we justify the existence of optimal solutions to $(P)$ by reducing it to the classical Weierstrass theorem in appropriate topological spaces.

Proof of Theorem 2.1. By the existence and uniqueness statements in Theorem 3.2 , there is a minimizing sequence $\left(y_{n}, u_{n}\right) \subset C\left([0, T] ; L^{2}(\Omega)\right) \times U_{a d}$ in problem $(P)$, where $y_{n}$ is the (unique) solution to (1.1) corresponding to $u_{n}$. Due to the growth condition in (A3), the sequence $\left(u_{n}\right)$ is bounded in $L^{2}(\Sigma)$. Thus we suppose without loss of generality that $\left(u_{n}\right)$ converges to $u$ in the weak topology of $L^{2}(\Sigma)$. Since $U_{a d}$ is closed and convex in (A4), one has $u \in U_{a d}$. It follows from the continuity statement in Theorem 3.2 that the sequence $\left(y_{n}, y_{n t}\right)$ is bounded in $L^{\infty}\left(0, T ; L^{2}(\Omega)\right) \times L^{\infty}\left(0, T ; H^{-1}(\Omega)\right)$, where $y_{n t}$ stands for the derivative of $y_{n}$. Employing the above continuity, we conclude that $\left(y_{n}, y_{n t}\right)$ converges to $\left(y, y_{t}\right)$ in the weak* topology of $L^{\infty}\left(0, T ; L^{2}(\Omega)\right) \times L^{\infty}\left(0, T ; H^{-1}(\Omega)\right)$, where $y$ is the solution to (1.1) corresponding to $u$. Invoking the closedness and convexity of $\mathcal{C}$ in (A4), one gets $y \in \mathcal{C}$. It remains to justify the lower semicontinuity of the cost functional

$$
J(y, u) \leq \liminf _{n \rightarrow \infty} J\left(y_{n}, u_{n}\right) \text { as } n \rightarrow \infty
$$

for the above weak convergences of $y_{n}$ and $u_{n}$. But this follows from the classical results on the lower semicontinuity of integral functionals with respect to the weak topologies under consideration due to the crucial convexity assumptions in (A1)-(A3). Thus $(y, u)$ is an optimal solution to the original optimal control problem $(P)$. 


\section{Adjoint System}

Our final goal is to prove the necessary optimality conditions formulated in Theorem 2.2. To proceed, we first need to clarify what we mean by solutions to the adjoint system in this theorem and then to establish some properties of adjoint trajectories allowing us to deduce the desired necessary optimality conditions for the hyperbolic control problem from an appropriate Lagrange Multiplier Rule for the auxiliary optimization problem in Banach spaces. Given $\left.\left.\mu \in \mathcal{M}_{b}(] 0, T\right] ; L^{2}(\Omega)\right)$, consider the system

$$
\begin{cases}p_{t t}-\Delta p=\left.\mu\right|_{Q} & \text { in } Q=\Omega \times] 0, T[ \\ p=0 & \text { on } \Sigma=\Gamma \times] 0, T[ \\ p(T)=0, \quad p_{t}(T)=-\left.\mu\right|_{\Omega \times\{T\}} & \text { in } \Omega,\end{cases}
$$

corresponding to $(2.3)$ with $\left(\lambda, y_{0}\right)=0$, where $\left.\mu\right|_{Q}$ (respectively to $\left.\mu\right|_{\Omega \times\{T\}}$ ) is the restriction of $\mu$ to $Q$ (respectively $\Omega \times\{T\}$ ). Observe that $\left.\mu\right|_{Q}$ belongs to the space $\mathcal{M}_{b}(] 0, T\left[; L^{2}(\Omega)\right)$, which is included in $\mathcal{M}_{b}(Q)$, and that $\left.\mu\right|_{\Omega \times\{T\}}$ belongs to $L^{2}(\Omega)$.

To define an appropriate notion of solutions to the adjoint system (4.1), suppose for a moment that $\left(p, p_{t}\right) \in L^{2}\left(0, T ; H_{0}^{1}(\Omega)\right) \times L^{2}\left(0, T ; L^{2}(\Omega)\right)$ and that $p_{t t}-\Delta p$, calculated in the sense of distributions on $Q$, belongs to $\mathcal{M}_{b}(Q)$. Then, following [13, Lemma 4.3] and using the divergence theorem, we can define the normal trace on $\operatorname{bd} Q$ for the vectorfield $\left(-\nabla p, p_{t}\right)$ as an element of $H^{-1 / 2}(\partial Q)$. Moreover, denoting this normal trace by $\gamma_{\nu_{Q}}\left(-\nabla p, p_{t}\right)$, we have the estimate

$$
\left\|\gamma_{\nu_{Q}}\left(-\nabla p, p_{t}\right)\right\|_{H^{-1 / 2}(\mathrm{bd} Q)} \leq C\left(\|p\|_{L^{2}\left(0, T ; H_{0}^{1}(\Omega)\right)}+\left\|p_{t}\right\|_{L^{2}(Q)}+\left\|p_{t t}-\Delta p\right\|_{\mathcal{M}_{b}(Q)}\right)
$$

where $C$ is independent of $p$. This allows us to define $p_{t}(0)$ as the restriction of this normal trace to $\Omega \times\{0\}$, i.e.,

$$
\left.\gamma_{\nu_{Q}}\left(-\nabla p, p_{t}\right)\right|_{\Omega \times\{0\}}=p_{t}(0) \in H^{-1 / 2}(\Omega) .
$$

Thus we come up to the following definition of weak solutions to the adjoint system given in (4.1).

Definition 4.1 (weak solutions to the adjoint system). A function $\left(p, p_{t}\right) \in$ $L^{\infty}\left(0, T ; H_{0}^{1}(\Omega)\right) \times L^{\infty}\left(0, T ; L^{2}(\Omega)\right)$ with $p_{t t}-\Delta p \in \mathcal{M}_{b}(Q)$ is a WEAK SOLUTION to 
the adjoint system (4.1) if one has the equality

$$
\begin{aligned}
& -\int_{\Omega} p(0) y_{1} d x+\left\langle p_{t}(0), y_{0}\right\rangle_{H^{-1}(\Omega) \times H_{0}^{1}(\Omega)} \\
& +\left\langle y\left(f, y_{0}, y_{1}\right), \mu\right\rangle_{\left.C\left([0, T] ; L^{2}(\Omega)\right) \times \mathcal{M}_{b}(00, T] ; L^{2}(\Omega)\right)}-\int_{Q} p f d x d t=0
\end{aligned}
$$

for all $\left(f, y_{0}, y_{1}\right) \in L^{2}(Q) \times H_{0}^{1}(\Omega) \times L^{2}(\Omega)$, where $y\left(f, y_{0}, y_{1}\right)$ denotes the unique solution to the homogeneous Dirichlet problem in (1.1), i.e.,

$$
\begin{cases}y_{t t}-\Delta y=f & \text { in } Q \\ y=0 & \text { on } \Sigma, \\ y(0)=y_{0}, \quad y_{t}(0)=y_{1} & \text { in } \Omega .\end{cases}
$$

Let us observe that, since $\left(p, p_{t}\right) \in L^{\infty}\left(0, T ; H_{0}^{1}(\Omega)\right) \times L^{\infty}\left(0, T ; L^{2}(\Omega)\right)$, one has $p \in C\left([0, T] ; L^{2}(\Omega)\right)$, and thus the term $\int_{\Omega} p(0) y_{1} d x$ is meaningful. Furthermore, $p_{t t}-$ $\Delta p \in \mathcal{M}_{b}(Q)$, and hence $p_{t}(0)=\left.\gamma_{\nu_{Q}}\left(-\nabla p, p_{t}\right)\right|_{\Omega \times\{0\}}$ is well defined in $H^{-1 / 2}(\Omega)$ due to the discussion right before the definition.

The next important result justifies the existence and uniqueness of weak solutions to the adjoint system (4.1) in the sense of Definition 4.1. Moreover, it provides additional regularity properties essential for the proof of the main theorem.

Theorem 4.2 (properties of adjoint arcs). The adjoint system (4.1) admits a unique weak solution $\left(p, p_{t}\right) \in L^{\infty}\left(0, T ; H_{0}^{1}(\Omega)\right) \times L^{\infty}\left(0, T ; L^{2}(\Omega)\right)$. Moreover, $p_{t}$ belongs to $B V\left([0, T] ; H^{-1}(\Omega)\right)$,

$$
\frac{\partial p}{\partial \nu}=\left.\gamma_{\nu_{Q}}\left(\nabla p,-p_{t}\right)\right|_{\Sigma} \quad \text { belongs to } L^{2}(\Sigma)
$$

p belongs to $C_{w}\left([0, T] ; H_{0}^{1}(\Omega)\right)$, and

$$
p_{t}(\tau) \text { belongs to } L^{2}(\Omega) \text { for all } \tau \in\{t \in[0, T] \mid \mu(\Omega \times\{t\})=0\} \text {. }
$$

In particular, one has $p_{t}(0) \in L^{2}(\Omega)$.

Proof. First observe that if $\left(p, p_{t}\right) \in L^{\infty}\left(0, T ; H_{0}^{1}(\Omega)\right) \times L^{\infty}\left(0, T ; L^{2}(\Omega)\right)$ satisfies (4.2) with $\mu=0$, then $p=0$. Thus system (4.1) admits at most one solution in the sense of 
Definition 4.1. We need to justify the existence of weak solutions with the additional regularity properties listed in the theorem.

Let $\left(\mu_{n}\right)$ be a sequence in $L^{1}\left(0, T ; L^{2}(\Omega)\right)$ satisfying the relations

$$
\begin{gathered}
\lim _{n \rightarrow \infty} \int_{Q} y \mu_{n} d x d t=\left\langle y,\left.\mu\right|_{Q}\right\rangle_{C\left([0, T] ; L^{2}(\Omega)\right) \times \mathcal{M}_{b}\left(\left[0, T\left[; L^{2}(\Omega)\right)\right.\right.} \text { for all } y \in C\left([0, T] ; L^{2}(\Omega)\right), \\
\int_{Q} \mu_{n} d x d t=\|\mu\|_{\mathcal{M}_{b}\left(j 0, T\left[; L^{2}(\Omega)\right)\right.}
\end{gathered}
$$

Denote by $p_{n}$ the (unique) solution to

$$
\begin{cases}p_{t t}-\Delta p=\mu_{n} & \text { in } Q \\ p=0 & \text { on } \Sigma \\ p(T)=0, \quad p_{t}(T)=-\left.\mu\right|_{\Omega \times\{T\}} & \text { in } \Omega .\end{cases}
$$

Employing the result of $[7$, Theorem 2.1], we have the estimate

$$
\begin{gathered}
\left\|p_{n}\right\|_{L^{\infty}\left(0, T ; H_{0}^{1}(\Omega)\right)}+\left\|p_{n t}\right\|_{L^{\infty}\left(0, T ; L^{2}(\Omega)\right)}+\left\|\frac{\partial p_{n}}{\partial \nu}\right\|_{L^{2}(\Sigma)}+\left\|p_{n}(0)\right\|_{H^{1}(\Omega)}+\left\|p_{n t}(0)\right\|_{L^{2}(\Omega)} \\
\leq C\|\mu\|_{\left.\mathcal{M}_{b}(0, T] ; L^{2}(\Omega)\right)}
\end{gathered}
$$

with a constant $C$ independent of $n$. It follows from (4.4) that the derivative of $p_{n t}$ with respect to $t$, in the sense of distributions on $Q$, can be represented in the form

$$
p_{n t t}=\pi_{n}+\mu_{n} \in L^{\infty}\left(0, T ; H^{-1}(\Omega)\right)+\mathcal{M}_{b}(] 0, T\left[; L^{2}(\Omega)\right) \subset \mathcal{M}_{b}(] 0, T\left[; H^{-1}(\Omega)\right)
$$

where $\pi_{n}$ is defined by

$$
\left\langle\pi_{n}, y\right\rangle_{L^{\infty}\left(0, T ; H^{-1}(\Omega)\right) \times L^{1}\left(0, T ; H_{0}^{1}(\Omega)\right)}:=\int_{Q} \nabla p_{n} \nabla y d x d t .
$$

Thus the sequence $\left(p_{n t t}\right)$ is bounded in $\mathcal{M}_{b}(] 0, T\left[; H^{-1}(\Omega)\right)$, and hence the corresponding one $\left(p_{n t}\right)$ is bounded in $B V\left([0, T] ; H^{-1}(\Omega)\right)$. Then there are $p \in L^{\infty}\left(0, T ; H_{0}^{1}(\Omega)\right)$ with $p_{t} \in B V\left([0, T] ; H^{-1}(\Omega)\right)$ and a subnet of $\left(p_{n}\right)$ that converges to $p$ in the weak* topology of $L^{\infty}\left(0, T ; H_{0}^{1}(\Omega)\right)$ and such that the corresponding derivatives $p_{n t}$ converge to $p_{t}$ in the weak* topology of $L^{\infty}\left(0, T ; L^{2}(\Omega)\right)$. Since the sequence $\left(\gamma_{\nu_{Q}}\left(-\nabla p_{n}, p_{n t}\right)\right.$ is bounded in $L^{2}(\partial Q)$, we may also suppose the convergence

$$
\gamma_{\nu_{Q}}\left(-\nabla p_{n}, p_{n t}\right) \rightarrow \gamma_{\nu_{Q}}\left(-\nabla p, p_{t}\right) \quad \text { weakly in } L^{2}(\partial Q)
$$


On the other hand, $\left.\gamma_{\nu_{Q}}\left(-\nabla p_{n}, p_{n t}\right)\right|_{\Omega \times\{T\}}=\left.\mu\right|_{\Omega \times\{T\}}$, and the sequence of

$$
\left.\gamma_{\nu_{Q}}\left(\nabla p_{n},-p_{n t}\right)\right|_{\Sigma}=\frac{\partial p_{n}}{\partial \nu}
$$

is bounded in $L^{2}(\Sigma)$. Thus

$$
\begin{aligned}
& \left.\left.\gamma_{\nu_{Q}}\left(\nabla p_{n},-p_{n t}\right)\right|_{\Sigma} \rightarrow \gamma_{\nu_{Q}}\left(\nabla p,-p_{t}\right)\right|_{\Sigma}=\frac{\partial p}{\partial \nu} \text { and } \\
& \left.\gamma_{\nu_{Q}}\left(-\nabla p_{n}, p_{n t}\right)\right|_{\Omega \times\{0\}}=\left.p_{n t}(0) \rightarrow \gamma_{\nu_{Q}}\left(-\nabla p, p_{t}\right)\right|_{\Omega \times\{0\}}=p_{t}(0)
\end{aligned}
$$

in the weak* topology of $L^{2}(\Sigma)$ and $L^{2}(\Omega)$, respectively.

Now passing to the limit as $n \rightarrow \infty$ in the equality

$$
\begin{aligned}
& -\left\langle p_{n}(0), y_{1}\right\rangle_{L^{2}(\Omega)}+\left\langle p_{n t}(0), y_{0}\right\rangle_{H^{-1}(\Omega) \times H_{0}^{1}(\Omega)} \\
& +\left\langle y\left(f, y_{0}, y_{1}\right), \mu_{n}\right\rangle_{\left.C\left([0, T] ; L^{2}(\Omega)\right) \times \mathcal{M}_{b}(00, T] ; L^{2}(\Omega)\right)}-\left\langle p_{n}, f\right\rangle_{L^{2}(Q)}=0
\end{aligned}
$$

we conclude that $\left(p, p_{t}\right)$ is the desired weak solution to the adjoint system (4.1) satisfying all but the last displayed relations in the theorem.

To prove the remaining property, we suppose that $\mu(\Omega \times\{t\})=0$ for some $t \in[0, T]$. Then considering the normal trace of $\left(-\nabla p, p_{t}\right)$ on $\partial(\Omega \times] 0, t[)$ as above, one gets that

$$
\left.\gamma_{\nu_{\Omega \times] 0, t[}}\left(-\nabla p, p_{t}\right)\right|_{\Omega \times\{t\}}=p_{t}(0) \in L^{2}(\Omega)
$$

which completes the proof of the theorem.

Finally in this section, we present a useful limiting consequence of Theorem 4.2 that ensures a Green-type relationship between solutions of the adjoint system (4.1) and the original arcs belonging to the space $Y$ of admissible state functions (3.3).

Theorem 4.3 (Green formula). Given $\left.\mu \in \mathcal{M}_{b}(] 0, T\right] ; L^{2}(\Omega)$ ), consider the unique solution $p$ to the adjoint system (4.1). Then for every admissible state function $y \in Y$, the adjoint arc $p$ satisfies the following Green formula

$$
\begin{aligned}
& \langle y, \mu\rangle_{\left.C\left([0, T] ; L^{2}(\Omega)\right) \times \mathcal{M}_{b}(00, T] ; L^{2}(\Omega)\right)}-\left\langle p, y_{t t}-\Delta y\right\rangle_{L^{\infty}\left(0, T ; H_{0}^{1}(\Omega)\right) \times L^{1}\left(0, T ; H^{-1}(\Omega)\right)} \\
& =-\int_{\Omega} y(0) p_{t}(0) d x+\left\langle y_{t}(0), p(0)\right\rangle_{H^{-1}(\Omega) \times H_{0}^{1}(\Omega)}-\int_{\Sigma} y \frac{\partial p}{\partial \nu} d s d t
\end{aligned}
$$

Proof. As proved in Theorem 4.2, the above Green formula holds for the solutions $p_{n}$ to the approximating adjoint system (4.4). Passing there to the limit as $n \rightarrow \infty$, we arrive at the required result (4.5). 


\section{Proof of Optimality Conditions}

This section is devoted to the proof of our main result formulated in Theorem 2.2. We employ the following strategy: reduce $(P)$ to a general optimization problem in Banach spaces for which necessary optimality conditions are known, and then express the latter optimization result and its assumptions in terms of the initial data of the original control problem $(P)$. This proof is essentially based on the specific results obtained in Section 4 for hyperbolic systems under consideration, which use in turn the regularity results of Theorem 3.2. The general optimization problem in Banach spaces, called the abstract control problem, is as follows:

$$
\inf \left\{I(z, \pi) \mid z \in Z, \pi \in \Pi_{a d}, G_{1}(z, \pi)=0, G_{2}(z) \in C_{2}\right\}
$$

where $Z$ is a Banach space, $\Pi$ is a separable Banach space, $\Pi_{a d}$ is a nonempty closed and convex subset of $\Pi, G_{1}$ is a mapping from $Z \times \Pi$ into a Banach space $Z_{1}, G_{2}$ is a mapping from $Z$ into a Banach space $Z_{2}$, and $C_{2}$ is a closed and convex subset in $Z_{2}$. As usual, we denote by $Z_{i}^{*}$ the topological dual of $Z_{i}$ for $i=1,2$ with the canonical duality pairing $\langle\cdot, \cdot\rangle$ on $Z_{i}^{*} \times Z_{i}$.

One can see that problem $(C P)$ involves infinite-dimensional operator constraints as well as geometric constraints given by convex sets. The variable $z$ and $\pi$ play a role of abstract state and control variables, respectively, relating to each other via the operator constraint $G_{1}(z, \pi)=0$. Necessary optimality conditions for general optimization problems of this type are known in the optimization theory. The following result given in [1] takes into account the specific structure of problem $(C P)$ and the convexity assumptions on the sets $\Pi_{a d}$ and $C_{2}$ that ensure a version of the Lagrange Multiplier Rule with maximization and minimization conditions over the convex sets involved in the problem.

Theorem 5.1 (necessary conditions for abstract control problems). Let $(\bar{z}, \bar{\pi})$ be an optimal solution to $(C P)$. Assume that $I$ is Fréchet differentiable at $(\bar{z}, \bar{\pi})$ while $G_{2}$ is Fréchet differentiable at $\bar{z}$, that $G_{1}$ is strictly differentiable at $(\bar{z}, \bar{\pi})$ with the surjective partial derivative $G_{1 z}^{\prime}(\bar{z}, \bar{\pi}): Z \rightarrow Z_{1}$, and that int $C_{2} \neq \emptyset$.

Then there are adjoint elements $(p, \mu, \lambda) \in Z_{1}^{*} \times Z_{2}^{*} \times \mathbb{R}^{+}$such that $(\lambda, \mu) \neq 0$ and 
the following conditions hold:

$$
\begin{gathered}
\lambda I_{z}^{\prime}(\bar{z}, \bar{\pi}) z+\left\langle p, G_{1 z}^{\prime}(\bar{z}, \bar{\pi}) z\right\rangle+\left\langle\mu, G_{2}^{\prime}(\bar{z}) z\right\rangle=0 \quad \text { for every } \quad z \in Z, \\
\left\langle\mu, z-G_{2}(\bar{z})\right\rangle \leq 0 \quad \text { for every } \quad z \in C_{2} \\
\lambda I_{\pi}^{\prime}(\bar{z}, \bar{\pi})(\pi-\bar{\pi})+\left\langle p, G_{1 \pi}^{\prime}(\bar{z}, \bar{\pi})(\pi-\bar{\pi})\right\rangle \geq 0 \quad \text { for every } \pi \in \Pi_{a d} .
\end{gathered}
$$

If in addition

$$
G_{1 z}^{\prime}(\bar{z}, \bar{\pi}) z_{0}+G_{1 \pi}^{\prime}(\bar{y}, \bar{w}) \pi_{0}=0 \quad \text { and } \quad G_{2}(\bar{z})+G_{2}^{\prime}(\bar{z}) z_{0} \in \operatorname{int} C_{2}
$$

for some $\pi_{0} \in\left(\Pi_{a d}-\bar{\pi}\right)$ and $z_{0} \in Z$, then the above conditions are fulfilled in normal form, i.e., with $\lambda=1$.

Now we complete the paper by proving the formulated necessary optimality conditions in the original control problem $(P)$.

Proof of Theorem 2.2. Let $(\bar{y}, \bar{u}) \in Y \times U_{a d}$ be the reference optimal solution to $(P)$. We are going to reduce $(P)$ to the $(C P)$ problem considering in Theorem 5.1. To furnish this, put:

$$
\begin{gathered}
Z=Y, \quad(z, \pi)=(y, u), \quad \Pi=L^{2}(\Sigma), \quad \Pi_{a d}=U_{a d}, \\
Z_{1}=L^{1}\left(0, T ; H^{-1}(\Omega)\right) \times L^{2}(\Sigma) \times L^{2}(\Omega) \times H^{-1}(\Omega), \quad Z_{2}=C\left([0, T] ; L^{2}(\Omega)\right), \quad C_{2}=\mathcal{C}, \\
I(y, u)=J(y, u), \quad G_{1}(y, u)=\left(y_{t t}-\Delta y-f,\left.y\right|_{\Sigma}-u, y(0)-y_{0}, y_{t}(0)-y_{1}\right), \quad G_{2}(y)=y .
\end{gathered}
$$

By assumptions (A5)-(A7) the cost functional $J$ is Fréchet differentiable at $(\bar{y}, \bar{u})$, the mapping $G_{1}$ is strictly differentiable at $(\bar{y}, \bar{u})$, and one has

$$
\begin{gathered}
J^{\prime}(\bar{y}, \bar{u})(y, u)=\int_{\Omega} \phi_{y}^{\prime}(x, \bar{y}(T)) y(T) d x+\int_{Q} g_{y}^{\prime}(x, t, \bar{y}) y d x d t+\int_{\Sigma} h_{u}^{\prime}(s, t, \bar{u}) u d s d t \\
G_{1}^{\prime}(\bar{y}, \bar{u})(y, u)=G_{1 y}^{\prime}(\bar{y}, \bar{u}) y+G_{1 u}^{\prime}(\bar{y}, \bar{u}) u, \quad G_{1 y}^{\prime}(\bar{y}, \bar{u}) y=\left(y_{t t}-\Delta y,\left.y\right|_{\Sigma}, y(0), y_{t}(0)\right) \\
G_{1 u}^{\prime}(\bar{y}, \bar{u}) u=(0,-u, 0,0) \quad \text { for every } \quad(y, u) \in Y \times L^{2}(\Sigma)
\end{gathered}
$$


Furthermore, it follows from Theorem 3.2 that the linear continuous operator $G_{1 y}^{\prime}(\bar{y}, \bar{u})$ is surjective from $Y$ to $L^{1}\left(0, T ; H^{-1}(\Omega)\right) \times L^{2}(\Sigma) \times L^{2}(\Omega) \times H^{-1}(\Omega)$. Thus all the assumptions of Theorem 5.1 are satisfied.

Applying the latter theorem, we find $\lambda \in \mathbb{R}^{+},(\bar{p}, \tilde{p}, \hat{p}, \breve{p}) \in L^{\infty}\left(0, T ; H_{0}^{1}(\Omega)\right) \times$ $L^{2}(\Sigma) \times L^{2}(\Omega) \times H_{0}^{1}(\Omega)$, and $\mu \in \mathcal{M}\left([0, T] ; L^{2}(\Omega)\right)$ with $(\lambda, \mu) \neq 0$ satisfying the following conditions:

$$
\begin{aligned}
& \int_{\Omega} \lambda \phi_{y}^{\prime}(x, \bar{y}(T)) y(T) d x+\int_{Q} \lambda g_{y}^{\prime}(x, t, \bar{y}) y d x d t+\left\langle\bar{p}, y_{t t}-\Delta y\right\rangle+\int_{\Sigma} \tilde{p} y d s d t \\
& +\langle\hat{p}, y(0)\rangle+\left\langle\breve{p}, y_{t}(0)\right\rangle+\langle\mu, y\rangle_{\mathcal{M}\left([0, T] ; L^{2}(\Omega)\right) \times C\left([0, T] ; L^{2}(\Omega)\right)}=0
\end{aligned}
$$

for every $y$ from the space of admissible state functions $Y$ in (3.3),

$$
\begin{gathered}
\langle\mu, z-\bar{y}\rangle_{\mathcal{M}\left([0, T] ; L^{2}(\Omega)\right) \times C\left([0, T] ; L^{2}(\Omega)\right)} \leq 0 \quad \text { for every } z \in \mathcal{C} \\
\int_{\Sigma}\left(\lambda h_{u}^{\prime}(x, \bar{y}, \bar{u})+\tilde{p}\right)(u-\bar{u}) d x \geq 0 \quad \text { for every } \quad u \in U_{a d}
\end{gathered}
$$

It follows from (5.2) and (A4) that $\left.\mu\right|_{\Omega \times\{0\}}=0$, and thus $\mu$ can be identified with a measure belonging to $\left.\left.\mathcal{M}_{b}(] 0, T\right] ; L^{2}(\Omega)\right)$. Furthermore, Theorem 4.2 ensures the existence of the unique weak solution $\left(p, p_{t}\right) \in L^{\infty}\left(0, T ; H^{1}(\Omega)\right) \times L^{\infty}\left(0, T ; L^{2}(\Omega)\right)$ to the adjoint system (2.3). Then the Green formula (4.5) of Theorem 4.3 and the optimality condition (5.1) yield that

$\left\langle p+\bar{p}, y_{t t}-\Delta y\right\rangle+\int_{\Sigma}\left(\tilde{p}-\frac{\partial p}{\partial \nu}\right) y d s d t+\int_{\Omega}\left(\hat{p}-p_{t}(0)\right) y(0) d x+\int_{\Omega}(\breve{p}+p(0)) y_{t}(0) d x=0$

for every $y \in Y$. Since the mapping $y \longrightarrow\left(y_{t t}-\Delta y,\left.y\right|_{\Sigma}, y(0), y_{t}(0)\right)$ is surjective from $Y$ to $L^{1}\left(0, T ; H^{-1}(\Omega)\right) \times L^{2}(\Sigma) \times L^{2}(\Omega) \times H^{-1}(\Omega)$, the above variational equality gives

$$
\begin{aligned}
& p=-\bar{p} \in L^{\infty}\left(0, T ; H_{0}^{1}(\Omega)\right), \quad \frac{\partial p}{\partial \nu}=\tilde{p} \in L^{2}(\Sigma), \\
& p_{t}(0)=\hat{p} \in L^{2}(\Omega), \quad \text { and } \quad p(0)=-\breve{p} \in H_{0}^{1}(\Omega) .
\end{aligned}
$$

Thus optimality conditions (5.1)-(5.3) of Theorem 5.1 imply the desired optimality condition (2.1) and (2.2) of Theorem 2.2 with the adjoint system (2.3). Observe finally that the qualification condition (2.4) of Theorem 2.2 reduces to the one in Theorem 5.1, which ensures the normality $\lambda=1$ and ends the proof of the theorem. 


\section{References}

[1] J. J. Alibert, J. P. Raymond, A Lagrange multiplier theorem for control problems with state constraints, Numer. Funct. Anal. Optim., 19 (1998), 697-704.

[2] N. Arada, J. P. Raymond, Optimality conditions for state-constrained Dirichlet boundary control problems, J. Optim. Theory Appl., 102 (1999), 51-68.

[3] N. Arada, J. P. Raymond, Dirichlet boundary control of semilinear parabolic equations, Part 2: Problems with pointwise state constraints, Appl. Math. Optim., 45 (2002), 145-167.

[4] E. Casas, Pontryagin's principle for state-constrained boundary control problems of semilinear parabolic equations, SIAM J. Control Optim., 35 (1997), 1297-1327.

[5] H. O. Fattorini, Infinite-Dimensional Optimization and Control Theory, Cambridge University Press, Cambridge, 1999.

[6] H. O. Fattorini, T. Murphy, Optimal control for nonlinear parabolic boundary control systems: the Dirichlet boundary conditions, Diff. Integ. Equ., 7 (1994), $1367-1388$.

[7] I. Lasiecka, J. L. Lions and R. Triggiani, Nonhomogeneous boundary value problems for second order hyperbolic operators, J. Math. Pures Appl. 65 (1986), 149192.

[8] I. Lasiecka and J. Sokolowski, Regularity and strong convergence of a variational approximation to a nonhomogeneous Dirichlet hyperbolic boundary problem, SIAM J. Math. Anal., 19 (1988), 528-540.

[9] I. Lasiecka and R. Triggiani, The regulator problem for parabolic equations with Dirichlet boundary control, Part 1: Riccati's feedback synthesis and regularity of optimal control, Appl. Math. Optim., 16 (1987), 147-168.

[10] X. J. Li and J. M. Yong, Optimal control theory for infinite-dimensional systems, Birkhäuser, Boston, 1995. 
[11] B. S. Mordukhovich, K. Zhang, Minimax control of parabolic systems with Dirichlet boundary conditions and state constraints, Appl. Math. Optim., 36 (1997), 323-360.

[12] B. S. Mordukhovich, K. Zhang, Dirichlet boundary control of parabolic systems with pointwise state constraints in International Conference on Control and Estimations of Distributed Parameter Systems, in Control and Estimation of Distributed Parameter Systems (Vorau, 1996), Intentational Series of Numerrical Mathematics, Vol. 126, Birkhäuser. Basel, 1998, pp. 223-236.

[13] J. P. Raymond, Nonlinear boundary control of semilinear parabolic problems with pointwise state constraints, Discrete Contin. Dynam. Systems, 3 (1997), 341-370.

[14] L. W. White, Control of a hyperbolic problem with pointwise stress constraints, J. Optim. Theory Appl., 41 (1983), 359-369.

[15] L. W. White, Distributed control of a hyperbolic problem with control and stress constraints, J. Math. Anal. Appl., 106 (1985), 41-53. 